\title{
Herramientas y métricas aplicadas al marketing editorial: del big data a la inteligencia artificial
}

Tools and metrics in editorial marketing: from big data to artificial intelligence

\author{
Antonia Salvador Benítez, Juan Miguel SÁnchez Vigil \\ Facultad de Ciencias de la Documentación (UCM), Santísima Trinidad, 37, 28010 Madrid, \\ asalvado@ucm.es,jmvigil@ucm.es
}

\begin{abstract}
Resumen
El sector editorial es un paradigma en lo que se refiere al marketing, con un espacio específico en las secciones generales del organigrama de las empresas, al que se suman: edición, producción, comercial, comunicación y derechos. La función del marketing es transversal y envolvente al interrelacionar con el resto de elementos de la cadena de elaboración del libro (impreso o digital). Son objeto de estudio en este artículo las herramientas y métricas aplicadas al marketing editorial, tomando como referente tres modelos (Tekstum, Jellybooks y Bookmetrix), que desde diferentes planteamientos, y con el big data y la inteligencia artificial soportando sus actividades, tienen el denominador común de conocer los hábitos de lectura a través del análisis de la información, al objeto de conseguir una mayor y mejor representación y organización del conocimiento que atraiga y fidelice lectores.
\end{abstract}

Palabras clave: Edición. Marketing editorial. Organización del conocimiento. Big data. Inteligencia artificial. Tekstum. Jellybooks. Bookmetrix.

\section{Introducción}

Big data e inteligencia artificial son términos cuyas aplicaciones llevan tiempo impulsando y transformando no solo cómo accedemos, interpretamos y procesamos datos. En el sector del libro se dispone de aplicaciones y dispositivos de lectura digitales que proporcionan una gran cantidad de datos sobre el comportamiento del lector. Tabletas y lectores electrónicos recopilan datos a través de las aplicaciones, lo que permite saber y medir, por ejemplo, los tiempos promedio de lectura, el momento de la misma o cómo se interactúa con ciertos géneros. Estos datos facilitan información para conocer y comprender el comportamiento de los lectores, lo que derivará en los modelos y contenidos de publicación, y en la identificación y recomendaciones personalizadas; en definitiva, en una mayor y mejor organización del conocimiento.

Son objeto de estudio en este artículo las herramientas y métricas aplicadas al marketing editorial, tomando como referente tres modelos (Tekstum, Jellybooks y Bookmetrix) que tienen el de-

\begin{abstract}
The publishing sector is a paradigm in terms of marketing with a specific space in the organization chart of the companies, to which Edition, Produc-tion, Commercial, Communication and Rights are added. The function of marketing is transversal and interrelates with the rest of the elements of the book production chain (printed or digital). The tools and metrics applied to editorial marketing are stud-ied in this article, taking as reference three models (Tekstum, Jellybooks and Bookmetrix), that from different approaches, and with big data and artificial intelligence supporting their activities, they have the common denominator of knowing reading habits through the analysis of information, in order to achieve a greater and better representation and organization of knowledge to attract and retain readers.

Keywords: Edition. Editorial marketing. Knowledge organization. Big data. Artificial Intelligence. Tekstum. Jellybooks. Bookmetrix.
\end{abstract}

nominador común de conocer los hábitos de lectura a través del análisis de la información. Para ello se examinan los parámetros e indicadores contemplados en cada caso a partir del testado y el análisis de los contenidos proporcionados en los website de cada una de las plataformas.

Sobre la planificación y el marketing en edición son varios los estudios de interés (Cole, 2003; Arqués Salvador, 2009; Gómez-Tarragona, 2010; Álvarez Ruiz, 2012; Escribano Hernández, 2012 y Fernández de la Puente-Campano, 2016), y en cuanto a métricas y big data se han tomado de referencia los clásicos (Valverde, 2016; González-Fernández, 2016, Zhao, Adib y Katabi, 2016; Serrano Cobos, 2014 y Barranco-Fragoso, 2012). Los datos de referencia relativos a la producción editorial, consumo y lectura proceden de la Panorámica de la edición de libros en España (Ministerio de Cultura) y de la Federación del Gremio de Editores de España.

Editores y autores disponen de perfiles en las redes sociales para gestionar y promover su presencia online e interaccionar con los lectores. 
También podemos ver esta tendencia en el crecimiento y el interés en colecciones digitales creadas cuidadosamente por los lectores en plataformas como Goodreads. Las acciones que derivan de estas revelaciones moldean e influyen en la creación de contenidos, así como en estrategias de marketing y comercialización con altas probabilidades de éxito. Los beneficios de estos datos son realmente positivos tanto para los lectores como para los editores: los primeros descubren más contenidos que responden mejor a sus necesidades, mientras que los segundos fomentan la creación de libros aún mejores, que a su vez generan beneficios al identificar aquello que interesa.

La tecnología permite convertir la información en inteligencia que tiende a redefinirse con la aparición de algoritmos. Estos datos pueden esconder y describir patrones de funcionamiento y relaciones, inapreciables a simple vista, pero que una vez tratados puede resultar una información muy útil como base para la toma de decisiones. El valor del big data surge cuando lo convertimos en información y, a través de la analítica, generamos información útil para la toma de decisiones.

Los datos masivos que aportan emails, encuestas, redes sociales, señales de móvil, conexiones, logins, entre otros muchos, ofrecen una información de altísimo valor, a veces incluso en tiempo real, para cualquier negocio, también para las editoriales. Chantal Restivo-Alessi, Directora Digital de Harper Collins, señala que la digitalización ha favorecido el acceso a los datos para conocer el comportamiento de los lectores, saber si se está llegando a la audiencia correcta, de la forma idónea y con los contenidos y formatos adecuados (Megapractical, 2016). A través de la venta directa, de las librerías online o mediante las plataformas sociales, el editor puede averiguar los gustos y hábitos de sus lectores, aspectos que le guiarán de manera efectiva en la gestión, desde el punto de vista de la estrategia de negocio, la promoción y el marketing.

Para ello es necesario definir qué queremos saber: hábitos, gustos, comportamientos, etc., es decir formular las preguntas estratégicas con cuyas respuestas, se puedan lograr los objetivos fijados. De este modo, las editoriales pueden testar y medir resultados, recabar otros nuevos y hacer los ajustes necesarios para evitar o reducir errores y alcanzar sus metas. Una de las principales aportaciones de los Big data es devolver a los clientes el valor de dicha información, ofreciéndoles servicios adaptados o productos totalmente nuevos que respondan mejor a sus intereses.
Prácticamente en cualquier área de una empresa o sector de actividad que nos imaginemos es posible encontrar aplicaciones para la analítica. En este artículo exploraremos de forma específica las relacionadas con el marketing en el sector editorial. Se presenta el estado actual del sector editorial, así como herramientas y tendencias de futuro que ponen en relación tres disciplinas que se van entrelazando a lo largo del texto: big data, o cómo y por qué se almacenan los datos y de dónde proceden; el papel de la Analítica de datos, o cómo extraer información útil de éstos mediante procesos matemáticos y estadísticos, y finalmente la inteligencia artificial, o cómo los datos pueden convertirse en inteligencia.

\subsection{No todo es big data}

Los big data figuran, junto con la impresión 3D, la inteligencia artificial, el desarrollo móvil o el Internet de las cosas, entre las principales tendencias tecnológicas dentro de la economía digital en los próximos cuatro años, según el informe anual de Gartner (2019). David Vivancos (2016) define big data como el "conjunto de tecnologías y procesos que están permitiendo capturar y almacenar cantidades ingentes de datos de distinta procedencia y tipologías, siendo la base tanto de la digitalización masiva del mundo analógico, como del almacenamiento de los propios datos generados en el mundo digital". Estos contenidos comenzaron siendo textos en chats y foros, luego fotografías y vídeos en redes sociales y blogs, y en los últimos años, se han sumado los datos de nuestro entorno. Así es como surgió el denominado Internet de las cosas (Internet of Things, IoT), donde millones de dispositivos transmiten sus datos de funcionamiento en tiempo real. Los big data poseen diferentes dimensiones que se complementan y que se denominan las 5 V's: Volumen, Velocidad, Variedad, Veracidad y Valor.

El valor de una empresa está también en los datos que posee, especialmente en aquellas cuya base de operaciones es Internet. La información y los datos siempre han estado ligados al poder, pero ahora esta afirmación cobra una dimensión mucho mayor. Los datos que generan las empresas son el factor diferencial -si se usan correctamente- para entender mejor a los clientes, optimizar los procesos y mejorar la competitividad. Sin embargo, no se considerarían big data por su volumen (según el tamaño de la empresa pueden oscilar de decenas de Megabytes a cientos de Gigabytes). Salvo en grandes empresas de comercio electrónico, no se usa un big data para almacenar los datos, que suelen estar gestionados a través de un software de CRM (Customer Relationship Management) o gestor de relaciones 
con los clientes, y por un software de ERP (Enterprise Resource Planning) o Planificador de Recursos. Tanto los CRM como los ERP suelen estar soportados por un gestor de bases de datos relacional que difiere de los usados por un big data, que trabaja con datos mucho más variados: textos, fotografías, vídeos, audio, series de datos temporales y muchos otros.

El marketing permite una visión global e integrada, que fusiona en tiempo real información de los CRM con los datos generados en los diversos canales de comunicación de la empresa, entre los que destacan los de las redes sociales. Así mismo pueden considerarse big data los contenidos audiovisuales, los datos de sensores, tanto del Internet de las cosas como de dispositivos wearables, de wikis y blogs de gran tamaño, gubernamentales, médicos, científicos, etc., en la mayoría de los casos con volúmenes de muchos Terabytes o Petabytes. Internet es el gran generador de contenidos y datos a mayor velocidad (Tabla I).

\begin{tabular}{ll}
\hline Contenido & Volumen/minuto \\
\hline Emails enviados & 188 millones \\
\hline $\begin{array}{l}\text { Mensajes } \\
\text { WhatsApp y Facebook } \\
\text { messenger) }\end{array}$ & 42 millones \\
\hline Vídeos Youtube & $\begin{array}{l}4.5 \text { millones videos } \\
\text { visualizados }\end{array}$ \\
\hline Búsquedas en Google & 4 millones \\
\hline Snapchat & $\begin{array}{l}2.1 \text { millones snaps } \\
\text { creados }\end{array}$ \\
\hline Netflix & 700 horas vistas \\
\hline
\end{tabular}

Tabla I. Internet in a minute, 2019 (Elaboración propia a partir de www.visualcapitalist.com)

\subsection{Analítica de datos e inteligencia artificial}

La medición es la clave de cualquier proyecto, y en el contexto del marketing digital, permite predecir el comportamiento del consumidor. En internet medimos consumo, comparación de contenidos, tráfico web, leads, ventas, etc.; sin embargo, lo más difícil no es obtener el dato, sino ser capaz de interpretarlo adecuadamente y tomar las decisiones oportunas. La analítica web es la evaluación y explicación racional de los datos, provenientes o no del big data, con objeto de tomar decisiones estratégicas a nivel de negocio, producto o precio, proponer mejoras o analizar tendencias y comportamientos recurrentes en el tiempo. Consistiría en formular una serie de preguntas con objeto de obtener y analizar los datos de una empresa, que pueden ser de dos tipos (Tabla 2):

(a) Datos de situación/informes. Ofrecen una visión del estado actual de una organización y forman parte del llamado business intelligence, con objeto de unificar los procesos de almacenamiento y tratamiento de la información generada en la actividad de una empresa, y utilizarlos como base para la toma de decisiones (1).

(b) Datos tácticos y estratégicos. Ofrecen un conocimiento más profundo de la empresa para poder estar preparados y anticipar las decisiones de futuro más inmediato. Permiten entender qué cambios son necesarios para alcanzar el mejor resultado posible. En estos datos tácticos y estratégicos es donde el retorno de la inversión o ROI (Return On Investment) (2) de la analítica predictiva es mucho mayor, en ocasiones cerca del $300 \%$.

En combinación con la analítica, el desarrollo de técnicas de visualización ha sido un aspecto fundamental para la interpretación de los datos, ya que precisamente, la mayor parte de nuestro cerebro está destinada al procesamiento de datos visuales.

\begin{tabular}{|c|c|c|}
\hline Tipos de datos & Objetivos & Preguntas clave \\
\hline $\begin{array}{l}\text { Datos de situación (Business } \\
\text { intelligence) }\end{array}$ & Conocer el estado actual de la empresa & ¿Qué ha sucedido? \\
\hline \multirow[t]{3}{*}{ Datos tácticos y estratégicos } & $\begin{array}{l}\text { Comprender los datos de la empresa para estar } \\
\text { preparados }\end{array}$ & ¿Por qué ha sucedido? \\
\hline & Anticipar las decisiones de futuro & ¿Qué será lo próximo que ocurrirá? \\
\hline & $\begin{array}{l}\text { Qué cambiar para obtener el mejor resultado } \\
\text { posible }\end{array}$ & $\begin{array}{l}\text { ¿Qué es lo mejor y lo peor que puede } \\
\text { suceder? }\end{array}$ \\
\hline
\end{tabular}

Tabla II. Analítica de datos (Business intelligence) 
El uso de gráficos $2 \mathrm{D} / 3 \mathrm{D}$, animaciones o incluso técnicas como la realidad virtual o aumentada están jugando un papel clave en el análisis de este tipo de datos, tan dinámico y multidimensional. De igual modo, ofrecen nuevas formas de presentar los resultados y métricas mediante animaciones y gráficos interactivos, que permiten al usuario modificar la visualización en función de los datos que le interesen.

Los algoritmos deep learning o de aprendizaje profundo de máquinas son los que han cosechado los mejores resultados en los últimos cinco años gracias principalmente a tres factores: la disponibilidad de cantidades ingentes de datos de diversas tipologías o un verdadero big data (contenidos de los que aprender); el crecimiento exponencial de la capacidad y velocidad de procesamiento de las máquinas (para aprender más rápido); y la aparición y desarrollo de una serie de algoritmos para simular el funcionamiento del cerebro humano dentro de una máquina, muchos de ellos ideados y descartados hace unas décadas, y que a día de hoy están demostrando ser excepcionalmente efectivos gracias a la existencia de los dos factores anteriores. En consecuencia, los puntos siempre se conectan a posteriori, y éste es el caso de las tres disciplinas que acabamos de introducir: el big data, la analítica y la inteligencia artificial.

\section{El sector editorial en cifras}

El sector editorial es una industria cultural que se organiza en torno a una cadena de valor cuyo producto final es el libro. Hasta ahora esta cadena era muy sencilla: autor, editor, productor, comercial y lector. Sin embargo, las nuevas tecnologías han alterado de forma radical la cadena, tanto en la producción como en el consumo al no haber un autor sino miles autores que escriben sus blogs o que autopublican gracias a la tecnología.

Internet ha reducido considerablemente el coste de creación de una editorial, y además es muy sencillo crear un espacio en la red, buscar autores dispuestos a escribir y vender sus creaciones. Lo que no ha cambiado es que sigue habiendo un lector, pero que ahora cuenta con infinidad de opciones para llegar a las lecturas y encontrar nuevos autores. El reto está, como antes, en la visibilidad.

El sector editorial ha tenido que hacer frente a un proceso de transformación como consecuencia de la irrupción digital. Este nuevo escenario ha multiplicado exponencialmente el número de fuentes a las que los lectores pueden acceder, surgiendo así un lector más participativo y más exigente. Ya no sólo se miden las ventas como dato último, sino la capacidad de los libros para convencer y entretener, es decir, el grado de compromiso del lector con cada título. De ahí que la personalización se haya convertido en un aspecto clave en la evolución de muchas empresas del sector editorial para afrontar una mayor y más fuerte competencia.

El sector del libro en España constituye un importante motor económico (mueve anualmente más de tres mil de millones de euros y emplea directa o indirectamente a más de 30.000 personas) posicionado entre las grandes potencias editoriales del mundo. Las cifras de la Panorámica de la edición de libros en España 2017 apuntan una tendencia positiva. El avance de Panorámica muestra descenso del 9,7\% en la producción editorial, pasando de 89.962 en 2017 a 81.228 ISBN en 2018. Esta bajada se refleja en la edición tanto de libros en papel como digitales. La producción de libros impresos disminuye un $1,1 \%$ con respecto a 2017 y representan el 74,9\% del total. La producción de libros impresos disminuye un $1,1 \%$, con un total de 60.835 libros. La edición en otros soportes ha disminuido el $28,3 \%$. La edición electrónica, incluida en la edición en otros soportes, desciende un $28,7 \%$, representando el $25,0 \%$. La mayor parte de los registros electrónicos (un 93,2\%) son libros digitales. Estos muestran un descenso del $31,4 \%$, representando el $23,3 \%$ del total de la producción. La mayor parte de los libros digitales se publican en formato Pdf, que suponen el $42,5 \%$, seguidos por ePub (33\%), Mobi $(580$ ISBN) y otros (4.054 ISBN). La edición de carácter público experimenta un descenso del 3,6\% y representa el $9,2 \%$ del total; mientras que la edición privada, con el $90,8 \%$ del total, disminuye un $10,3 \%$. El $24,8 \%$ de la producción privada fue editada por 90 empresas editoriales, que representan el 2,9\% de las que tuvieron actividad en 2018.

Según el informe del Comercio Interior del Libro elaborado por el Gremio de Editores, la facturación en 2017 fue de 2.319,36 millones de euros, apenas un $0,1 \%$ más que en 2016 y sigue sostenido por los libros de papel siendo las librerías y cadenas de librerías los principales canales de venta de libros, representando más de la mitad de la facturación total $(52,8 \%)$. La facturación del libro digital representa el $5,1 \%$ del total del sector, con un incremento del 1,6\% respecto al año anterior. En cuanto a los géneros literarios, crecieron todos, tanto en adulto $(1,0 \%)$ como en infantil $(3,2 \%)$. Además crecen los libros científico-técnicos y universitarios (un $4,3 \%$ más), los de ciencias sociales y humanidades $(2,2 \%)$, los libros prácticos $(3,3 \%)$ y los cómics $(4,2 \%)$. Los libros de divulgación general se mantuvieron, mientras que los diccionarios y enciclopedias descendieron. No obstante, la facturación se concentra en 
empresas de Barcelona (50,8\%) seguida de Madrid $(42,3 \%)$.

La proliferación de sellos independientes es, junto a la concentración en grandes grupos como Planeta o Penguin Random House, la tendencia de las últimas décadas. Según el Observatorio de la Lectura y el Libro cada semana nacen de media en España seis nuevas editoriales.

\section{Marketing editorial: herramientas y métricas}

El análisis de datos en las empresas puede aportar valor a los usuarios (descuentos, experiencias formativas, entretenimiento, soluciones, apps, etc.). Empresas como TripAdvisor, Netflix o Amazon, gracias al big data y a la analítica, ofrecen promociones, paquetes descuento y precios dinámicos basados en datos, sistemas de recomendación que ayudan a entender mejor a los clientes, personalizar la oferta y mejorar la competitividad de las empresas.

La agregación de datos se revela como otro factor a considerar: navegación móvil, geolocalización, conexión e incluso el modo de teclear pueden ofrecer información valiosa sobre los consumidores. Sin embargo, la heterogeneidad y los diferentes perfiles de usuarios (hábitos, gustos, etc.) hacen que la información no sea uniforme. En este sentido, la agregación de datos de diferentes comportamientos y perfiles puede favorecer una mayor optimización para aumentar las ventas.

El conocimiento de los lectores y sus hábitos de consumo y lectura de libros constituyen importantes datos que las editoriales pueden gestionar para mejorar la oferta de servicios y contenidos para dirigirse a sus potenciales audiencias. La clave está en conocer qué datos sería necesario recopilar. Así mismo es importante identificar qué tipo de información dispersa puede ofrecer una nueva visión junto con los datos globales. Compartir dichos datos también puede ayudar a que los diferentes eslabones de la cadena mejoren sus márgenes de actuación de manera interna a la hora de pensar en las ventas a potenciales lectores.

Joe Wikert (2015), especialista en estrategia y desarrollo de negocios, ofrece un método para acotar la recogida de información sobre los hábitos de lectura y propone una batería de cuestiones clave: ¿Cuántas personas abren el e-book que han comprado? ¿Han leído el e-book de manera lineal o saltando páginas o capítulos? ¿En qué punto se abandona la lectura? ¿Cuál es la tasa de conversión de los capítulos gratuitos a la compra del e-book? ¿Qué horas del día y qué días de la semana son los más habituales para la lectura? ¿Cuánto tiempo se dedica a la lectura de las citas/notas al pie? ¿Se llega a consultar el índice o tabla de contenidos? ¿Qué frases se subrayan, comentan y comparten con mayor frecuencia?

A estas preguntas se han incorporado otras también importantes, que completan los objetivos anteriores: tipo de formato elegido; origen de la plataforma de compra; dispositivo de lectura; app móvil preferida para leer y horarios de compra online.

Junto a los datos a los que pueden acceder las editoriales desde sus propios sistemas de venta y difusión, en los últimos años están surgiendo otras plataformas y herramientas anexas para ampliar el conocimiento del lector y conseguir una mejor definición de lo que quieren o buscan los potenciales lectores. Algunos de los datos que se pueden registrar son el tipo de dispositivo, horario y frecuencia de lectura, tasa de abandono, lugar del libro donde se abandona la lectura, además de todas las valoraciones y comentarios que comparten los lectores sobre un libro. De cómo los editores utilicen estas herramientas para interactuar con el lector, y de cómo interpreten y aprovechen todo este big data dependerá su éxito.

\subsection{Tekstum: La experiencia emocional de la} lectura como base de la recomendación

Ideada por los economistas Marc Santandreu y Marc Martínez y apoyada por el Ministerio de Industria, Energía y Turismo, Tekstum es una potente plataforma de recomendación de libros basada en los big data que analiza la experiencia emocional que resulta de la lectura de un libro utilizando la inteligencia artificial y las opiniones, valoraciones y comentarios de los lectores en blogs, redes sociales y plataformas literarias. Con toda esta información, el equipo de Tekstum ha creado un diccionario de más de 20.000 palabras específicas del mundo editorial para identificar los sentimientos que provoca una obra. El análisis de estos datos permite descubrir la experiencia real de los lectores, entender por qué les gusta un libro e identificar oportunidades de venta.

Tekstum está dirigida tanto a lectores, escritores, editores y empresas relacionados con la industria editorial, así como agentes literarios, bibliotecarios $u$ otros profesionales que trabajen en o con este sector. Permite optimizar tanto la venta de los libros como la venta de experiencias, centrándose tanto en las necesidades de los lectores como en las necesidades de los editores. El sistema proporciona un análisis científico en tiempo real de las opiniones, comentarios y reseñas que 
los lectores realizan en plataformas literarias, blogs y redes sociales, que van más allá de simples listas de los más vendidos, metadatos editoriales o puntuaciones con estrellas que no contemplan los matices que pudieran existir entre ellas. Opera en las redes y aprovecha los datos utilizando la información contextual de comentarios y tweets para ofrecer información cualitativa del mercado del libro a los distintos agentes del sector, mejorar los resultados de las recomendaciones y fortalecer la proporción de éxitos de editores, autores, ejecutivos de marketing y ventas. Con este objetivo proporciona información relacionada con los libros y su impacto en el mercado que va desde un análisis de la popularidad y el sentimiento que despierta un libro (3), un perfil del autor y la medición de su reputación digital, basado en el número de seguidores en Twitter o Facebook; las consultas realizadas en Wikipedia sobre un título específico, las personas más influyentes en las redes sociales (Top Influencers) así como el origen, sexo y edad de los lectores o audiencias potenciales, entre otros aspectos.

El informe incluye algunos datos sobre el análisis emocional y el impacto del libro, información sobre el autor y su popularidad, su número de seguidores en Twitter o "Me gusta" en Facebook y la media de páginas vistas al mes en la Wikipedia.

El acceso mediante registro ofrece la misma información que la versión gratuita pero abierta a la selección de libros que desee el usuario, que puede solicitar el análisis de un libro introduciendo algunos datos (por ejemplo: autor, título o ISBN), de modo que el informe se genera de forma automática bajo demanda.

Puede realizarse el análisis de ocho libros al mes, sin perjuicio de que los interesados puedan acordar el análisis de un mayor número de libros. El modelo de informe incluye la siguiente información: Puntuación media del análisis emocional, reputación del autor, tasa de retención o de impacto social (Top of mind), popularidad, noticias o conceptos relacionados con el libro analizado en la red (Sensación de nube), información sobre el autor, número de seguidores en Twitter, número de "Me gusta" en Facebook, visitas a la página de Wikipedia, demografía de los lectores, género, edad, ubicación, y "Top Influencers" en Twitter, es decir, personas de referencia relacionadas con el libro en las redes sociales. También se puede conocer el número de visualizaciones en Youtube y menciones en otras plataformas de medios de comunicación (prensa, radio, televisión) así como una comparativa entre libros similares o relacionados para contrastar datos.
Las cifras que ha acumulado esta plataforma ponen de relieve el impacto que ha tenido en el sector (134.021.533 de libros publicados hasta 2016; 50.000 .000 reseñas en plataformas literarias, 6.000 tweets/segundo y 4.000 .000 millones de likes/minuto en Facebook en 2016) con clientes recurrentes que generan (Grupo Planeta, Strands, Sinerrata y The Spanish Bookstage que generan tracción (4).

El Big Data emocional de Internet nos descubre que libros están triunfando. Se miden las emociones positivas, negativas o neutras que está despertando cualquier publicación. Y su evolución a lo largo de un año. A partir de aquí se crea una nube de palabras que se convierte en el ADN emocional del libro. Una vez estos datos han sido tratados, los editores los pueden usar para decidir los próximos lanzamientos. Además, ha diseñado un nuevo método de recomendación de libros. Actualmente el lector puntúa y se guía a través de los libros más vendidos, pero el que propone Tekstum es un recomendador $100 \%$ emocional. Marc Santandreu, cofundador de Tekstum comenta (Trinidad, 2015):

¿Qué es la Literatura? subjetividad, emociones, sentimientos y eso las ventas no lo dicen. Por eso nosotros buscamos esta información subjetiva y la convertimos en datos objetivos para que los editores puedan tomar decisiones, como si reeditan un libro o no, o cómo enfocar la campaña de marketing.

Según un estudio de la compañía norteamericana Deloitte, las consultas hechas desde dispositivos móviles influyen un $64 \%$ en las ventas de libros en los establecimientos de los Estados Unidos. El objetivo de Tekstum es que su aplicación se utilice para consultar los sentimientos que despierta un libro justo antes de que los lectores realicen una compra (5).

\subsection{Jellybooks y el compromiso de los lectores}

Jellybooks (https://www.jellybooks.com), empresa londinense pionera en analizar el modo en que leemos y la interacción del lector con el libro, ha desarrollado proyectos para la recuperación de información editorial, por ejemplo: si se ha leído el libro, los dispositivos elegidos para leer, horarios, frecuencia de lectura, tiempo en terminarlo, en qué punto se ha abandonado la lectura o si el lector lo recomienda, entre otros aspectos. El objetivo es entender mejor la conexión entre el lector y el libro, y detectar de un modo más directo qué títulos son los más adecuados para cada tipo de lector.

Para la recopilación de estos datos de lectura JeIlybooks ha desarrollado un software inteligente (candy.js) incrustado en libros electrónicos, especialmente en formato ePub. El procedimiento 
para la recogida de datos es través de campañas en las que se invita a participar a una muestra de lectores (entre 200 y 500 personas) a los que se informa que van recibir un libro electrónico con un software de seguimiento integrado para registrar sus datos de lectura. Hay dos tipos de campañas: dirigidas al análisis de un solo título, o campañas multitítulo en la que se ofrece a los lectores una selección de la que pueden elegir dos títulos.

En la mayoría de los casos, se trata de libros que aún no han sido publicados. En otros se someten a prueba libros en tapa dura para edición de bolsillo; o bien, cuando los editores quieren decidir sobre la edición de una secuela de un libro o similar. Conviene precisar que todos los libros distribuidos por Jellybooks (copias pre-pub, fragmentos o ejemplares gratuitos) están sujetos a derechos de autor y no está permitido copiar, imprimir o compartir. Cada documento tiene un identificador único y el software de seguimiento añade marcas de agua digitales que permiten seguir el rastro del libro y evitar acciones de piratería y descarga ilegal.

La lectura puede realizarse con aplicaciones compatibles (iBooks, Ebook Reader, Adobe Digital Editions, Bookshelf, Azardi y Cloudshelf Reader) (6), y la duración de una campaña oscila entre cuatro y seis semanas, periodo que se registra si el lector "abre" el libro, si lo termina, si lo lee en una sola sesión o en varias, el horario y frecuencia de lectura, el dispositivo utilizado y la forma en que avanza o se desplaza por los contenidos.

La tasa de libros abandonados -y el punto en que se abandona su lectura- es un dato importante para la editorial, ya que puede saber qué es lo que hace que la historia, llegada a un punto, deje de funcionar. También los hábitos de lectura constituyen un indicador.

El compromiso con un libro es uno de los requisitos previos que motiva a los lectores a recomendar un título. De acuerdo con una Encuesta de Nielsen Global Trust In Advertising, el 92\% de los usuarios tiene en cuenta las recomendaciones de amigos o familiares, lo que las convierte en fuentes de confianza con una gran influencia para los consumidores (Nielsen, 2016). También recurren a expertos y líderes para obtener orientación para facilitar el proceso de toma de decisiones. Según el estudio Anual de Redes Sociales (IAB España, 2018 ), el $72 \%$ de los usuarios de redes sociales entre 16 y 55 años en España siguen perfiles de personas que marcan tendencias y lideran opiniones, es decir, los denominados influencers.

Las redes sociales se han convertido en el nuevo boca a boca, el lugar donde los usuarios comparten sus opiniones y experiencias con un producto determinado, sin embargo The Keller Fay Group -agencia especializada en interacción, y social media para grandes marcas- ha demostrado que solo el $10 \%$ del boca a boca se produce online. Para lo demás se siguen utilizando los contactos directos, donde herramientas privadas como WhatsApp han capitalizado la conversación social relevante (Fernández de la Puente-Campano, 2016). En consecuencia, la medición de la tasa de terminación y el compromiso del lector se convierten en posibles indicadores para la previsión de qué título podría construir un fuerte boca a boca Rhomberg (2015). Lo interesante de esta herramienta de monitorización es que, a diferencia de Google Analytics, puede trabajar offline, de este modo los datos son capturados y almacenados localmente hasta que el usuario se conecta a Internet y envía los datos recopilados con un solo click.

La medición y analítica web es fundamental para Jellybooks, ya que proporciona un análisis basado en datos para medir el compromiso del lector con la mayor rapidez y, por tanto, la comercialización y la campaña de publicidad pueden ser ejecutadas con el máximo efecto. En este sentido es ante todo una herramienta de marketing para autores y editores.

La analítica dirigida a los autores mide cómo los lectores se involucran con los libros (compromiso), su satisfacción y su probabilidad de recomendarlo a los demás. Pone a prueba un libro antes y después de su publicación, a través de grupos de discusión virtuales, copias de lectura avanzada o libros gratuitos. También mediante el seguimiento de lectura de fragmentos, para detectar si el lector se engancha y compra una copia completa.

En el caso de los editores la analítica proporciona conocimientos aplicables a las necesidades de la audiencia para tratar de evitar errores: cómo gestionar los escasos recursos para publicidad y promoción; cómo optimizar el título, la descripción y presentación del libro; y dónde posicionarlo en términos de audiencia para lograr un fuerte boca a boca. Editores expertos como Random House, Elsevier, MacMillan y Bonnier ya están haciendo uso de estas herramientas de medición para aumentar sus ventas y la cuota de mercado de sus títulos. En la mayoría de los casos estos editores utilizan la analítica antes de que el libro sea publicado para decidir sobre los gastos de marketing y dónde enfocar los recursos de promoción y publicidad.

Jellybooks es la primera y hasta ahora única empresa que permite a los editores recoger los datos de aplicaciones de terceros para entender el comportamiento de los lectores, a diferencia de Amazon, Apple y Google, que no comparten los datos 
que recogen. Trabaja con tres indicadores clave de rendimiento (KPI) aplicados al sector editorial: Tasa de finalización de la lectura, Factor de recomendación y Factor de ajuste de la cubierta.

(a) Tasa de finalización de la lectura: Porcentaje de lectores que inician y finalizan un libro. Es uno de los KPI básicos para medir la satisfacción del lector con el contenido y se calcula dividiendo el número de lectores que terminaron el libro entre el número total de lectores que envió la lectura del libro en cualquier punto o capítulo del libro. Generalmente, si los lectores no terminan una novela, es que su grado de satisfacción no era muy grande. Las tasas de finalización no parece que sean dependientes del género. Un resultado por debajo del $25 \%$ indica un compromiso débil; entre $25-50 \%$ es un buen resultado y se logra en la mayoría de los libros; y un resultado superior al $50 \%$ es muy bueno, especialmente para los de no ficción, e indica que un libro destaca por encima del resto. Una tasa por encima del $75 \%$ es excepcional, cifra que sólo han alcanzado un $5 \%$ de los libros analizados por Jellybooks, siendo algunos de ellos los mayores éxitos de ventas de los últimos años.

(b) Factor recomendación: Este indicador es una adaptación del concepto Net Promoter Score (NPS) y consiste en preguntar a los lectores si recomendarían el libro a sus amigos. Las cuatro respuestas posibles son: "Sin duda lo recomendaría a un amigo" (estos serían los promotores); "Posiblemente lo recomendaría a un amigo" (neutrales); "Probablemente no se lo recomendaría a un amigo" (detractores débiles) y "Sin duda no lo recomendaría a un amigo" (detractores fuertes). Para calcular este KPI sólo se considera la respuesta de los lectores que han terminado el libro, y se obtiene restando el porcentaje de detractores débiles y fuertes, siendo el resultado el número de promotores. Un libro con factor de recomendación positiva tiene más promotores que detractores. Un porcentaje entre el $30 \%$ y el $60 \%$ sigue siendo un resultado muy bueno, mientras que un título con más del $60 \%$ de promotores tiene el potencial de un hit viral. Como venimos señalando, el factor de recomendación es un indicador clave del potencial del boca a boca de un libro. Libros con altas tasas de finalización y alto factor de recomendación son los principales candidatos para una fuerte campaña de marketing y relaciones públicas.

(c) Factor de ajuste de la cubierta: La cubierta es una de las razones más comunes por las que un contenido tiene que vender. Al igual que ocurre con el diseño de los carteles de cine -en los que convergen los intereses dispares del director, el productor y el distribuidor- en el diseño de la cubierta de un libro también se produce esta tensión entre las propuestas del autor y el editor. Para el autor y el lector, la portada evoca el contenido y lo sintetiza. En las condiciones de saturación que definen el actual mercado editorial, el principal mérito de la cubierta es la diferenciación, tanto en la mesa de novedades como en la plataforma de venta online. Este indicador permite conocer si, en opinión de los lectores, el contenido del libro coincide con la expectativa planteada en la cubierta.

Todos estos datos son procesados, agregados y analizados y se presentan en gráficos interactivos con diferentes códigos de color para identificar si los resultados son pobres (azul), buenos (amariIlo), muy buenos (verde) o excelentes (púrpura). Este método de visualización facilita la interpretación a cualquier usuario, ya sea un editor, un experto en marketing o un gestor de medios sociales. Se trata de datos inteligentes no de grandes datos, que alcanzan su potencial máximo cuando se comparten con el equipo y el autor.

\begin{tabular}{lll}
\hline Tipo de datos & Fuente & Métrica Bookmetrix \\
\hline Citas & Springer & $\begin{array}{l}\text { Número de citas/año } \\
\text { Listado de trabajos que citan el libro/capítulo } \\
\text { Actualización semanal }\end{array}$ \\
\hline Menciones & Altmetric & $\begin{array}{l}\text { Número total de menciones online/propias del libro/capítulo } \\
\text { Actualización en tiempo real }\end{array}$ \\
\hline Lectores & Mendeley & $\begin{array}{l}\text { Datos demográficos y ubicaciones geográficas } \\
\text { Actualización semanal }\end{array}$ \\
\hline Reseñas & Springer & $\begin{array}{l}\text { Número de reseñas del libro seleccionado } \\
\text { Actualización semanal }\end{array}$ \\
\hline Descargas & Springer & $\begin{array}{l}\text { Número total de descargas de capítulos } \\
\text { Actualización mensual }\end{array}$ \\
\hline
\end{tabular}

Tabla III. Datos, fuentes y métricas de la plataforma Bookmetrix 
3.3. Bookmetrics y el impacto de las publicaciones científicas

Bookmetrix (www.bookmetrix.com) es un proyecto de la editorial Springer Science + Bussines Media Altmetric que reúne un conjunto de métricas de rendimiento para buscar, conocer y comparar el impacto de las publicaciones científicas.

Ofrece una visión global del alcance, uso y número de lectores de libros o capítulos sueltos proporcionando métricas a diferentes niveles: citas, menciones en línea, estadísticas de lectores, reseñas y descargas. Bookmetrix recupera los metadatos de los libros y capítulos de Springer y CrossRef y agrega las métricas asociadas a dichos libros y capítulos a partir de diversas fuentes (Tabla III).

Los informes son públicos y fácilmente accesibles e incluyen la información del libro, un listado de todos los capítulos y la visualización de las métricas asociadas al libro y/o capítulos.

\section{Neuromarketing y tendencias editoriales}

Junto a los grandes volúmenes de datos cargados de racionalidad, lo cierto es que en la mayoría de las decisiones de compra los argumentos basados en la lógica suelen quedar en segundo plano ante los mecanismos emocionales. En los últimos tiempos se están generalizando aproximaciones a las técnicas de persuasión como el neuromarketing, que trata de explicar que el comportamiento del consumidor no responde a normas ni pautas razonables, sino que adopta expresiones muy complejas basadas en la emocionalidad.

Una de las grandes aliadas en este contexto ha sido la neurociencia, puesto que un mejor conocimiento del funcionamiento del cerebro humano puede ayudar a entender mejor las verdaderas necesidades y gustos del consumidor, y a comunicarnos mejor con él. De ahí su desarrollo y aplicación en el marketing (neuromarketing) y la comunicación (neurocomunicación).

El Laboratorio de Inteligencia Artificial del Instituto de Tecnología de Massachusetts (MIT), elaboró EQ-Radio (http://eqradio.csail.mit.edu), dispositivo que puede revolucionar estas prácticas. Se trata de una herramienta que puede utilizar las señales wireless para detectar nuestro estado emocional con la misma precisión que un cardiograma, midiendo la variabilidad del pulso cardiaco y la respiración. Este dispositivo determina con un $87 \%$ de probabilidad si una persona está feliz, triste, enfadada, etc. Los datos son ejecutados mediante una serie de algoritmos y procesados por una máquina de auto-aprendizaje que busca parámetros que coincidan con el comportamiento de una persona que siente una emoción específica.

Con ella se pretende humanizar la interacción de los usuarios con la tecnología con la mayor precisión posible. Se espera que facilite la labor a todo tipo de sectores -también al editorial- a la hora de determinar qué quieren los consumidores, no sólo por sus gustos e intereses sino también, en función de su estado de ánimo. Por el momento EQ-Radio necesita la participación voluntaria de los usuarios, pero su desarrollo es una evidencia más de las posibilidades que ofrece la tecnología para profundizar en el conocimiento de las personas, clientes potenciales de cualquier servicio o producto.

En consecuencia, una webcam podría bastar para que mientras leemos un libro, asistimos a un concierto online o miramos una obra de arte, la tecnología sepa mediante nuestros gestos si nos gusta o no, y qué sensación nos produce. Esta tecnología se utiliza a través de cámaras de vigilancia para saber la satisfacción de sus clientes, aplicable a las librerías para conocer en tiempo real las emociones predominantes de los visitantes: reacciones a cubiertas de libros, precios, experiencias, servicios, sonido e iluminación para favorecer el bienestar, mejorar la experiencia de compra y aumentar las ventas. Todo para lograr cierta estimulación y también para la anticipación.

Art19 (art19.com), es una compañía de podcasts que proporciona servicios a editores y anunciantes para dirigirse a las audiencias creando mensajes de audio personalizados y ayudando a los editores a rentabilizar estas grabaciones gracias al análisis del comportamiento del usuario. Otra experiencia piloto es a2o (www.a2o.santillana.com), que aprovecha la potencia de los datos para ofrecer experiencias de aprendizaje personalizadas en el ámbito docente.

En un mercado competitivo donde la personalización es la clave - y no es posible personalizar sin datos personales- el empleo de las tecnologías (redes sociales, aplicaciones móviles, e-commerce, etc.) y la interacción con los clientes resulta indispensable para la estrategia empresarial (7). Sin embargo, el mismo usuario que demanda productos y servicios personalizados, con frecuencia se escandaliza si las empresas le trackean (8) para ofrecerle la personalización que él mismo solicita, y lo que resulta aún más grave, reconoce que no lee las políticas de privacidad (Salvador Benítez, 2019).

En España, la nueva Ley Orgánica 3/2018, de 5 de diciembre, de Protección de Datos y Garantía 
de los Derechos Digitales (LOPD GDD) -conocida como LOPD 2018- da cobertura al Reglamento General de Protección de Datos (RGPD), de obligado cumplimiento en todos los países de la Unión Europea. En el caso de España -donde la protección de datos es un derecho fundamental protegido por el artículo 18.4 de la Constitución- el RGPD incorpora novedades, tanto en el régimen de consentimiento y tratamiento de los datos como en la introducción de nuevas figuras y procedimientos (Salvador Benítez, 2019).

\section{Conclusiones}

De la investigación y análisis sobre el tema propuesto, se han obtenido las conclusiones que se indican:

Los avances tecnológicos y el abaratamiento del almacenamiento junto con el desarrollo de las técnicas estadísticas están permitiendo extraer conocimiento derivado del análisis de datos masivos procedentes de distintas fuentes, con un coste mucho menor y con mayor riqueza y exactitud para la toma de decisiones. En su mayoría se trata de datos personales, asociados a las operaciones que los consumidores llevan a cabo en cualquier sector, incluido el editorial, Nos encontramos inmersos en la economía del dato y términos como big data o inteligencia artificial se han vuelto cotidianos en los planes estratégicos de cualquier organización, independientemente de la industria de la que se trate.

El big data aplicado al sector editorial es una "fuente" para generar información que, analizada y gestionada, permite conocer la penetración en los diferentes mercados, la fijación de precios, las posibilidades de éxito de autores noveles, etc. Gracias a los datos masivos y la analítica, las empresas ofrecen precios dinámicos basados en datos, y sistemas de recomendación que cada vez conocen más al consumidor y pueden personalizar la oferta.

En edición, el control de recursos hace necesaria una inversión en análisis de datos. Teniendo en cuenta que una de las principales características de los big data es la velocidad, la posibilidad de rastrear el comportamiento e información de los potenciales lectores de forma rápida y eficiente permite reducir los riesgos. Más allá de disponer de una u otra solución tecnológica, para llevar a cabo una buena estrategia de analítica, es importante que las organizaciones se rodeen de un capital humano apto para esta tarea. Entre los perfiles clave destacan el Científico de datos (Data Scientist) en la parte más especializada, y el CDO (Chief Data Officer) o Jefe de Datos, en la parte más estratégica.
A nadie escapa que la retención masiva de datos de carácter socioeconómico, demográfico, financiero y de comportamiento con propósitos analíticos puede tener graves consecuencias para la privacidad y libertad individual de los consumidores. Desde una perspectiva de seguridad y privacidad, la normativa reguladora, la tecnología que sustenta las plataformas y las empresas, se enfrentan al reto de garantizar que los usuarios tengan el suficiente control sobre sus datos para prevenir un uso indiscriminado a la vez que se mantiene su utilidad para extraer conocimiento, patrones y, en suma, valor como activo empresarial.

La cadena lineal de valor del libro en la que intervienen autor, agente, editor, impresor, distribuidor, librero, crítico y lector se ha sustituido por una red de valor, con relaciones más fluidas entre los eslabones. Las nuevas formas relacionales en el sector parecen tender a generar redes en relación al contenido más que a la posición en la cadena de valor, que se sustituye por una red de valor. En un entorno en el que todos podemos publicar contenidos autoeditados (denominados non traditional publishing), las editoriales dejan de tener el control sobre la producción y se convierten fundamentalmente en mediadores culturales multimedia que explotarán los contenidos en todos los formatos a su alcance. En consecuencia, las herramientas como las que aquí se analizan pasan a ser decisivas en la toma de decisiones.

La materialidad del libro físico ha dado paso al contacto personal y la interacción e intercambio de opinión con otros lectores. El poder de la prescripción es cada vez mayor y la recomendación es un extraordinario motor de venta de libros, quizá el principal. Para que podamos recomendar una lectura, el contenido debe provocar emociones que nos empujen a hablar del libro con otros y lo conviertan en un objeto social. Nos dirigimos hacía una web cada vez más personal, que se adapta a lo que busca el usuario, que aprende de las páginas que visita, que sirven para alimentar la inteligencia colectiva. Las preferencias que el usuario haya reconocido como propias son la base para recomendar qué libros tiene que leer o qué lugares tiene que visitar alguien que está conectado con él.

\section{Notas}

(1) El business intelligence no se debe confundir con el Big Data, ya que podría no serlo, dependiendo del volumen y de otros criterios.

(2) El ROI es uno de los principales medidores por los que se rigen las actividades de una empresa. Aunque en ocasiones es difícil de medir, trata de relacionar la inversión 
realizada para poner en marcha una actividad determinada y los resultados económicos que ha generado.

(3) El sistema utiliza un algoritmo propio basado en tecnología big data e inteligencia artificial que descubre las emociones y sentimientos que un libro transmite a sus lectores. Para ello se enseña una máquina a identificar e interpretar diferentes sentimientos dentro de un texto utilizando técnicas de Procesamiento del Lenguaje Natural (PLN). Este método se denomina "Análisis de Sentimiento" y permite identificar y clasificar automáticamente la opinión de un texto como positivo, negativo o neutro (es decir, que no contiene ningún sentimiento). Es capaz de clasificar automáticamente el sentimiento porque busca patrones ocultos en grandes volúmenes de datos cualitativos y luego usa esta información para aprender características comunes en el uso del lenguaje que indican un sentimiento específico. Por lo tanto, cada patrón es indicativo de un sentimiento particular: consisten de patrones gramaticales, tendencias lexicales, tonalidad, uso de vocabulario particular, Coloquialismos, frases, etc. Al aprender de estos patrones textuales, se puede predecir la opinión de un texto desconocido debido a sus similitudes con otros contextos indicativos de un sentimiento específico.

(4) Si hay un factor importante para una startup es la tracción. Se trata de una métrica del progreso que demuestra la validación real de un modelo de negocio, cómo está siendo aceptado por los clientes y si hay una demanda real en el mercado. En definitiva, la tracción es un elemento clave para tomar nuevas decisiones sobre el futuro de la compañía y para buscar inversores que hagan crecer el proyecto. La base es tener un buen producto que cubra problemas reales de los clientes.

(5) Las aplicaciones no compatibles con Jellybooks son: Kindle, Nook, Kobo, Overdrive Mediaconsole, Tolino, Aldiko, Google Play Books y Moon+Reader.

(6) En la actualidad la plataforma Tekstum no se encuentra activa por falta de financiación. El análisis y testado se realizó durante el periodo que permaneció operativa.

(7) La gestión de la información de los usuarios constituye quizá el aspecto más desconocido, controvertido e incierto de las redes y plataformas sociales. La investigación sobre la materia ha generado varios trabajos entre los que señalamos el estudio realizado por Inteco y la Agencia Española de Protección de Datos (2009); Salvador Benítez y Gutiérrez David (2010); Díaz Buck (2013); Roura y Ferré (2014), Salvador, Olivera y Sánchez (2014), Salvador Benítez (2019).

(8) El término se refiere básicamente a seguir puntualmente los "movimientos" de los usuarios. Las analíticas de Google agregan este elemento denominado Heatmaps.

\section{Referencias}

Álvarez Ruiz, Antón (2012). La magia del planner. Cómo la planificación estratégica puede potenciar la comunicación persuasiva. Madrid: ESIC.

Arqués Salvador, Neus (2009). Marketing para escritores. Cómo publicar, promocionar y vender tu libro. Barcelona: Alba editorial.

Barranco-Fragoso, Ricardo (2012). ¿Qué es big data? IBM. http://www.ibm.com/developerworks/ssa/local/im/que-esbig-data (2020-01-20).

Cole, David (2003). Marketing editorial. La guía. México: Fondo Nacional para la Cultura y las Artes.

Díaz Buck, Anid Vanessa (2013). La autorregulación en redes sociales como forma de garantizar los derechos de intimi- dad, privacidad y protección de datos personales. // Derecom. ISSN: 1988-2629. 13 (marzo-mayo 2013), 125143.

Escribano Hernández, Asunción (2012). La retórica publicitaria editorial. El arte de vender un libro. Madrid: Arco Libros

Fernández de la Puente-Campano, Jaime (2016). Plan de marketing digital. Claves para triunfar. Madrid: The Valley Digital Business School.

Gartner, Inc. (2019). Top 10 Strategic Technology Trends for 2020. A Gartner Special Report. https://www.gartner.com/en/doc/432920-top-10-strategic-technologytrends-for-2020. (2020-01-20)

Gómez-Tarragona, Daniel (2010). Marketing editorial. Cómo satisfacer las necesidades de los lectores de libros. Madrid: Ediciones Pirámide

González-Fernández Villacencio, Nieves (2016). Métricas de la web social para bibliotecas. Barcelona: UOC.

González, Manuel (2017). Tekstum, el mejor analista de libros. https://www.elreferente.es/innovadores/tekstum101 innovadores-30616 (2020-01-20).

Instituto Nacional de Tecnologías de la Comunicación; Agencia Española de Protección de Datos. (2009). Estudio sobre la privacidad de los datos personales y la seguridad de la información en las redes sociales online. https://www.csirtcv.gva.es/sites/all/files/downloads/estudio_intecoaepd_privacidad_redes_sociales.pdf (202005-29)

España (2018). Ley Orgánica 3/2018, de 5 de diciembre, de Protección de datos personales y garantía de los derechos digitales // BOE. 294 (6-12-2018), 119788-119857. https://www.boe.es/eli/es/lo/2018/12/05/3 (2020-05-29)

Megapractical (2016). Cómo crear valor en el sector editorial a través de los Big Data. https://www.megapractical.com/noticias/como-crear-valor-en-el-sector-editoriala-traves-de-los-big-data (2020-01-20).

Nielsen Global Trust In Advertising (2016). Winning Strategies for an evolving Media Landscape. https://www.nielsen.com/wp-content/uploads/sites/3/2019/04/globaltrust-in-advertising-report-sept-2015-1.pdf (2020-01-20)

Panorámica de la edición de libros en España 2017 (2018). Madrid: Secretaría General Técnica. Centro de Publicaciones. Ministerio de Educación, Cultura y Deporte.

Rhomberg, Andrew (2015). Jellybooks: Tracking Reader Engagement for Better Marketing. // Publishing perspectives. (August 26, 2015). https://publishingperspectives.com/2015/08/jellybooks-tracking-reader-engagement-for-better-marketing/

Roura, Elisabeth; Ferré Pavia, Carme. (2014). Aspectos legales de las redes sociales: protección de datos y uso consciente de la tecnología. // Ferré Pavia, C. (Ed.) (2014). El uso de las redes sociales: ciudadanía, política y comunicación. La investigación en España y Brasil. Universitat Autónoma de Barcelona: Institut de la Comunicació, 92115.

Salvador Benítez, Antonia (2019). Entre la privacidad y el negocio. Condiciones de uso y política de datos en redes sociales // Derecom. ISSN: 1988-2629. 27 (SeptiembreMarzo 2019), 139-161.

Salvador Benítez, Antonia; Gutiérrez David, Estrella. (2010). Redes sociales y medios de comunicación. Desafíos legales. // El Profesional de la Información, ISSN: 16992407. 19:6 (Noviembre-Diciembre 2010) 667-674.

Salvador Benítez, Antonia; Olivera Zaldua, Maria; Sánchez Vigil, Juan Miguel (2014). Redes sociales y medios de comunicación. Políticas de uso de contenidos audiovisuales. // Sierra Sánchez, Javier; García García, Francisco (2014). Tecnología y narrativa audiovisual. Madrid: Fragua, 717-735. 
Serrano Cobos, Jorge (2014). Big data y analítica web. Estudiar las corrientes y pescar en un océano de datos. // EI profesional de la información. ISSN: 1699-2407. 23:6 (Noviembre-Diciembre 2014), 561-565.

Trinidad, Meritxell. (2015). Un sistema para medir la emoción que provoca un libro. elmundo.es (04-06-2015). https://www.elmundo.es/economia/2015/06/02/556c9bab 22601d913e8b4591.html (2020-01-20).

Valverde, Jaime (2016). Redes sociales. Cómo diseñar una estrategia de éxito. Madrid: The Valley Digital Business School.

Vivancos, David (2016). Big Data: Hacia la inteligencia artificial. Madrid: The Valley Digital Business School.
Wikert, Joe (2015). [Blog]. Here's how reader analytics can help publishers. https://jwikert.typepad.com/the_average joe/2015/06/heres-how-reader-analytics-can-helppublishers.html (2020-01-20).

Zhao, Mingmin.; Adib, Fadel.; Katabi, Dina. (2016). Emotion recognition using wireless signals. // Chen, Yingying; Gruteser, Marco (2016). MobiCom'16. The 22nd Annual International Conference on Mobile Computing and Networking. New York: Association for Computing Machinery, 95-108. http://eqradio.csail.mit.edu/files/eqradio-paper.pdf (2020-01-20).

Enviado: 2020-03-29. Segunda versión: 2020-06-04. Aceptado: 2020-06-05. 